Case

\title{
Recurrent Aortic Aneurysm due to Behcet's Disease: A Case Report from China
}

\author{
Zhongchen Zhang, MD, Xiangdong Jian, MD, PhD, Huimin Liu, MD, Wei Zhang, MD, and \\ Qian Zhou
}

\begin{abstract}
In this report, we present a twenty-nine year old patient with a five-year history of Behcet's disease (BD), who developed an ascending aortic aneurysm. Chest enhanced computed tomography scanning demonstrated a saccular aneurysm of the ascending aorta, with a maximum diameter of $11 \mathrm{~cm}$. The patient was administered with prednisone thalidomide and anticoagulant. A regular follow up was carried out. The patient lived well with blood sedimentation and $\mathrm{C}$-reactive protein in the normal range.
\end{abstract}

Keywords: Behcet's disease, ascending aortic aneurysm, thalidomide, anticoagulation

\section{Introduction}

Behcet's disease (BD) is a systemic autoimmune disease characterized by major symptoms of oral aphthous ulcers, genital ulcers, uveitis and skin lesions. The frequency of vascular involvement is about $8 \%,{ }^{1)}$ among which aneurysm is rare. We described a male with a five years history of BD, who developed an ascending aortic aneurysm.

\section{Case Report}

The patient was a twenty-nine year old male, whose primary syndrome was a vascular lesion. Enhanced computed tomography scanning demonstrated that the patient had a brachiocephalic trunk pseudoaneurysm five years ago (Fig. 1). Surgery was performed under cardiopulmonary bypass and gerenal anaesthesia. Pseudoaneurysm exclusion was initiated and then bypass from the ascending aorta to common carotid artery with an artificial

Department of Poisoning and Occupational Disease, Qilu Hospital of Shandong University, Jinan, Shandong, China

Received: February 9, 2012; Accepted: March 30, 2012

Corresponding author: Xiangdong Jian, MD, PhD. Department of Poisoning and Occupational Disease, Qilu Hospital of Shandong University, No. 107, Road Wenhuaxi, Jinan, 250012 Shandong, China Email: j6anxian@public.jn.sd.cn

(C)2012 The Editorial Committee of Annals of Thoracic and Cardiovascular Surgery. All rights reserved. prosthetic graft was utilized. No pathological examination was performed. Cervical vascular murmur disappeared after the surgery. Three years later before admission to our department, he was worried about recurrent oral and genital ulcers. Physical examination showed multiple ulcers on mouth mucosa and skin of scrotum and swelling in the superior extremity of the chest; the diameter of the basilar part was about $5 \mathrm{~cm}$, and the cutaneous pathergy test was positive. Blood examination showed a white blood cell count, red blood cell count, and hemoglobin, all in the normal range; blood sedimentation was $35 \mathrm{~mm}$ per hour, and C-reactive protein was $208 \mathrm{mg}$ per liter. The blood culture was negative. Chest enhanced computed tomography scanning demonstrated that the patient has a saccular aneurysm on the ascending aorta with a diameter of $6 \times 9 \times 11 \mathrm{~cm}$. The aneurysm showed a big cavity, combined with thrombosis (Figs. 2 and 3).

The diagnosis relied upon the criteria proposed by the International Study Group for Behcet's disease. Because of poor economical conditions, the patient refused a second operation and accepted medicinal therapy. Medicinal therapy included oral prednisone $30 \mathrm{mg}$ once per day, thalidomide $100 \mathrm{mg}$ once per night, aspirin $100 \mathrm{mg}$ once per day, aceclofenac $50 \mathrm{mg}$ twice per day. Prednisone was decrement gradually to $10 \mathrm{mg}$ per day as the mucosa ulcer improved. A regular follow-up was carried out strictly for eighteen months. The patient is asymptomatic and lives well now. He comes to our outpatient room, once a month. 


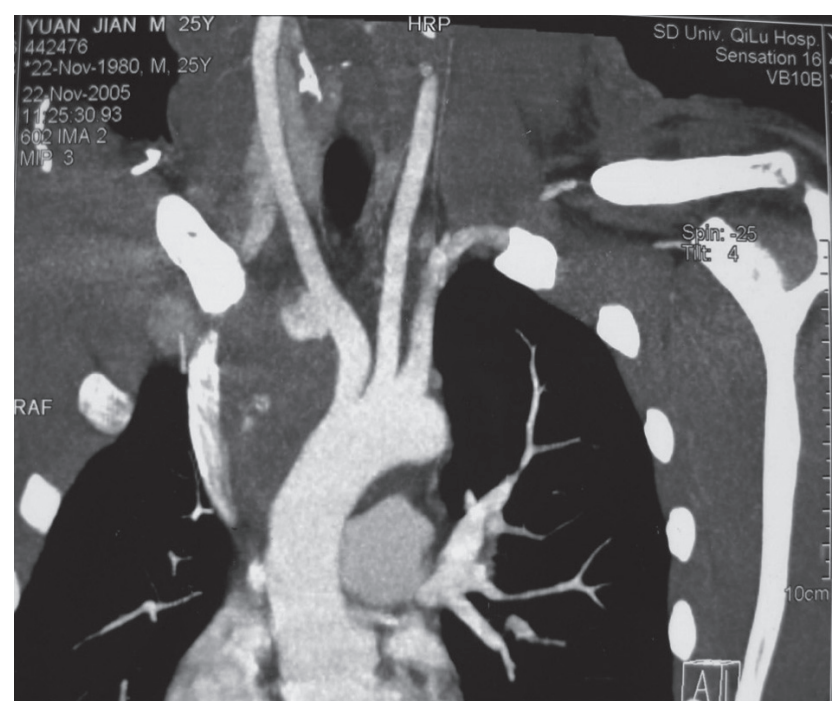

Fig. 1 Arteriography of brachiocephalic trunk pseudoaneurysm of the case in 2005.

\section{Discussion}

Behcet's syndrome was first reported by a dermatologist in 1937.') In 1961, Mishima first described an aortic aneurysm in a 38-year-old man with $\mathrm{BD}$, and since then, have seen a patient with a femoral artery aneurysm. ${ }^{3)}$ Aneurysm was one of the most severe symptom manifestations and had a poor prognosis. It was associated with a high mortality observed in BD. Davatchi had summarized 6500 cases in Iran, the frequency of aneurysm was $0.5 \%$. Saadoun showed that the main cause of death in BD patients was major vessel disease, mainly arterial aneurysm and Budd-Chiari syndrome, about 43.9\% ${ }^{4)}$ Medicine and surgery therapy would be better to controlling the symptom.

Thalidomide was first introduced in 1957 as sedative agent and antiemetics in the German pharmaceutical market. ${ }^{5)}$ In 1990s, it was began to used to treat rheumatologic diseases, such as BD. Thalidomide was found to decrease tumor necrosis factor-alpha level and also inhibit angiogenesis. ${ }^{6,7)}$ Sayarlioglu found that thalidomide reduced both tumor necrosis factor and neutrophil migration, which did better to mucocutaneous lesion of $\mathrm{BD}^{8)} \mathrm{In}$ our case, Thalidomide $100 \mathrm{mg}$ was given once per night. No adverse reaction appeared.

In 2006, Vahedian described a case of pseudoaneurysm associated with BD in a 17-year-old boy with a recurrent right ventricular thrombus. Postoperatively, anticoagulation therapy was administrated, the thrombus of the right ventricle disappeared 4 months later. ${ }^{9)}$ Herein,

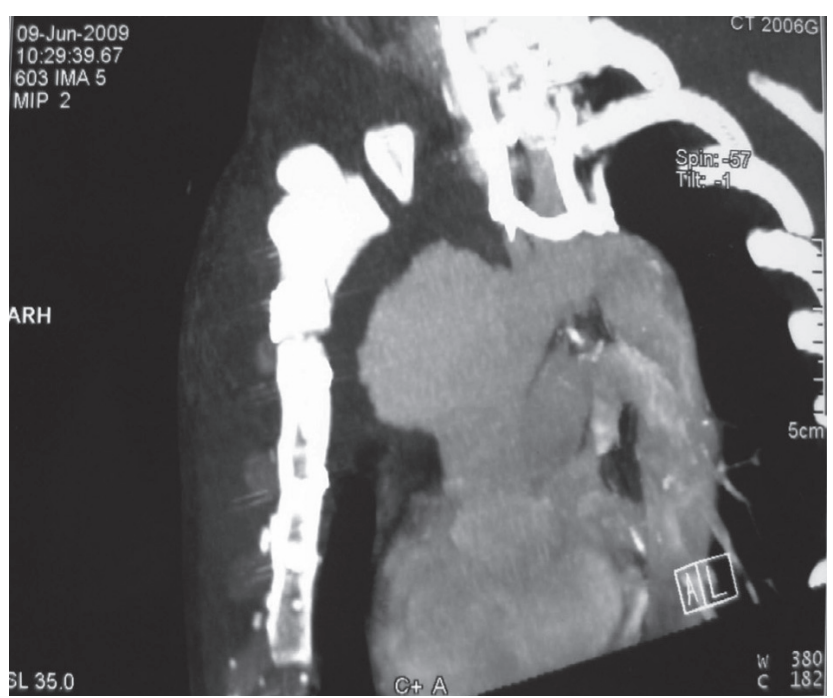

Fig. 2 Arteriography of saccular aneurysm on ascending aorta in 2009.

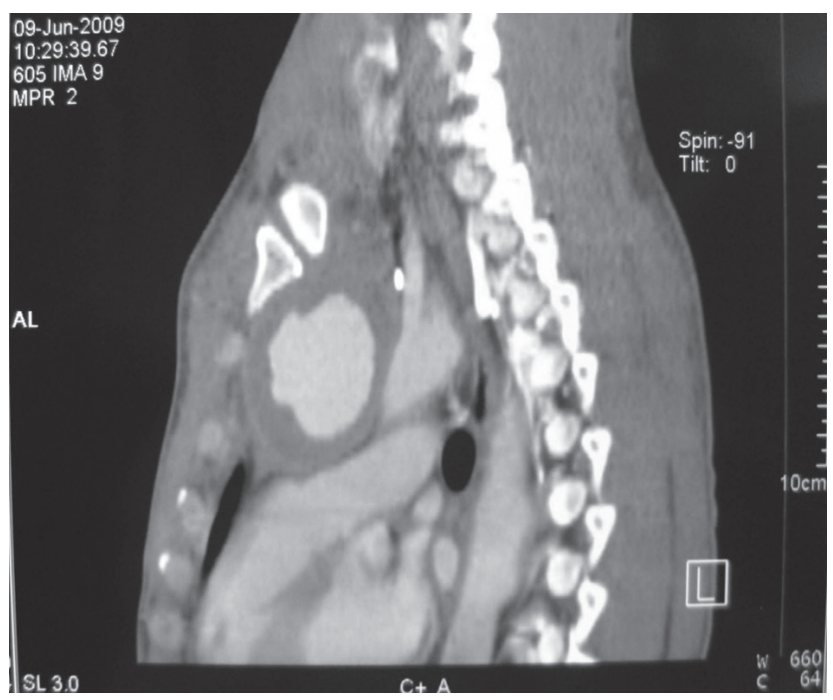

Fig. 3 Arteriography of saccular aneurysm on ascending aorta and bulging of chest in 2009 .

our patient received aspirin $100 \mathrm{mg}$ per day constantly no bleeding was found. Swelling at the superior extremity of the sternum turned smaller, three months later.

Non-steroidal anti-inflammatory drugs could inhibit cyclooxygenase and decrease prostaglandins level in vivo. Prednisone had a strong anti-inflammatory effection, and also inhibited cellular immunity. The patient maintained minimum dosage.The patient was asymptomatic after six month medicine therapy. An intensive follow up would be kept on. 


\section{Conclusion}

We presented a patient with a five-year history of BD, who developed an ascending aortic aneurysm. This case suggests that medicine therapy is effective in treating fatal aneurysm of BD.

\section{Disclosure Statement}

The authors declare that there are no conflicts of interest.

\section{Reference}

1) Davatchi F, Shahram F, Chams-Davatchi C, et al. Behcet's disease in Iran: analysis of 6500 cases. Int J Rheum Dis 2010; 13: 367-73.

2) Kojima N, Sakano Y, Ohki S, et al. Rapidly growing aortic arch aneurysm in Behcet's disease. Interact Cardiovasc Thorac Surg 2010; 21.
3) Hills EA. Behçet's syndrome with aortic aneurysms. Br Med J 1967; 4: 152-4.

4) Saadoun D, Wechsler B, Desseaux K, et al. Mortality in Behçet's disease. 2010; 62: 2806-12.

5) Yasui K, Uchida N, Akazawa Y, et al. Thalidomide for treatment of intestinal involvement of juvenile-onset Behçet disease. Inflamm Bowel Dis 2008; 14: 396400.

6) Direskeneli H, Ergun T, Yavuz S, et al. Thalidomide has both anti-inflammatory and regulatory effects in Behcet's disease. Clin Rheumatol 2008; 27: 373-5.

7) Calabrese L, Resztak K. Thalidomide revisited: pharmacology and clinical applications. Expert Opin Investig Drugs 1998; 7: 2043-60.

8) Sayarlioglu M, Kotan MC, Topcu N, et al. Treatment of recurrent perforating intestinal ulcers with thalidomide in Behçet's disease. Ann Pharmacother 2004; 38: 808-11.

9) Vahedian J, Sadeghpour A. Arterial homograft and medical therapy in pseudoaneurysm of infrarenal aorta concomitant with recurrent right ventricular thrombus in Behcet's disease. Saudi Med J 2006; 27: 1401-3. 